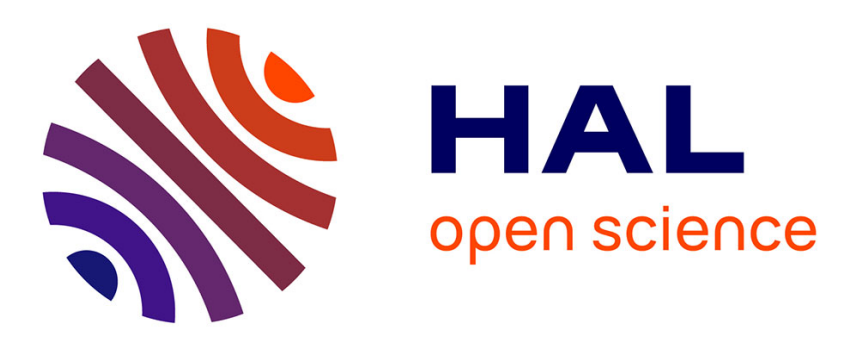

\title{
The isotropic remnant of the CO2 near-fully depolarized Raman 2v3 overtone
}

\author{
Michel Chrysos, I.-A. Verzhbitskiy, Florent Rachet, A.-P. Kouzov
}

\section{To cite this version:}

Michel Chrysos, I.-A. Verzhbitskiy, Florent Rachet, A.-P. Kouzov. The isotropic remnant of the CO2 near-fully depolarized Raman 2v3 overtone. Journal of Chemical Physics, 2011, 134 (10), Non spécifié. 10.1063/1.3557820 . hal-03344647

\section{HAL Id: hal-03344647 \\ https://univ-angers.hal.science/hal-03344647}

Submitted on 15 Sep 2021

HAL is a multi-disciplinary open access archive for the deposit and dissemination of scientific research documents, whether they are published or not. The documents may come from teaching and research institutions in France or abroad, or from public or private research centers.
L'archive ouverte pluridisciplinaire HAL, est destinée au dépôt et à la diffusion de documents scientifiques de niveau recherche, publiés ou non, émanant des établissements d'enseignement et de recherche français ou étrangers, des laboratoires publics ou privés. 


\title{
A The Journal of Chemical Physics
}

\section{The isotropic remnant of the $\mathrm{CO} 2$ near-fully depolarized Raman $2 \mathrm{v} 3$ overtone}

\author{
M. Chrysos, I. A. Verzhbitskiy, F. Rachet, and A. P. Kouzov
}

Citation: The Journal of Chemical Physics 134, 104310 (2011); doi: 10.1063/1.3557820

View online: http://dx.doi.org/10.1063/1.3557820

View Table of Contents: http://scitation.aip.org/content/aip/journal/jcp/134/10?ver=pdfcov

Published by the AIP Publishing

\section{Articles you may be interested in}

More light on the 2v5 Raman overtone of SF6: Can a weak anisotropic spectrum be due to a strong transition anisotropy?

J. Chem. Phys. 140, 034308 (2014); 10.1063/1.4861047

Molecular dynamics simulations for $\mathrm{CO} 2$ spectra. IV. Collisional line-mixing in infrared and Raman bands J. Chem. Phys. 138, 244310 (2013); 10.1063/1.4811518

The isotropic spectrum of the CO2 Raman 2v3 overtone: A line-mixing band shape analysis at pressures up to several tens of atmospheres

J. Chem. Phys. 134, 224301 (2011); 10.1063/1.3596750

The depolarized Raman 2v3 overtone of $\mathrm{CO} 2$ : A line-mixing shape analysis

J. Chem. Phys. 134, 194305 (2011); 10.1063/1.3580278

Are asymmetric stretch Raman spectra by centrosymmetric molecules depolarized?: The $2 \mathrm{v} 3$ overtone of $\mathrm{CO} 2$ J. Chem. Phys. 134, 044318 (2011); 10.1063/1.3535599

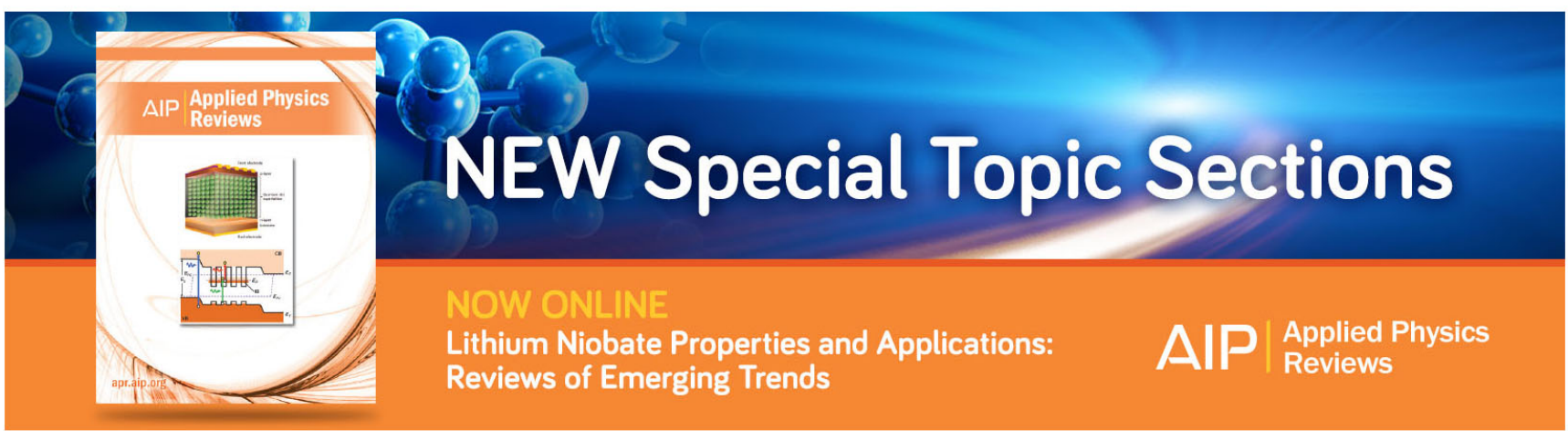




\title{
The isotropic remnant of the $\mathrm{CO}_{2}$ near-fully depolarized Raman $2 v_{3}$ overtone
}

\author{
M. Chrysos, ${ }^{1}$ a) I. A. Verzhbitskiy, ${ }^{1}$ F. Rachet, ${ }^{1}$ and A. P. Kouzov ${ }^{2}$ \\ ${ }^{1}$ MolTech-Anjou, UMR CNRS 6200, Université d'Angers, 2 boulevard Lavoisier, 49045 Angers, France \\ ${ }^{2}$ Institute of Physics, Saint Petersburg State University, Ulyanovskaya str. 1, Peterhof, Saint Petersburg 198504, \\ Russia
}

(Received 24 December 2010; accepted 1 February 2011; published online 14 March 2011)

\begin{abstract}
In a recent paper [M. Chrysos, I. A. Verzhbitskiy, F. Rachet, and A. P. Kouzov, J. Chem. Phys. 134, 044318 (2011)], we showed that, in $\mathrm{CO}_{2}$, the $2 v_{3}$ transition generates a Raman line spectrum that is 98\% depolarized, a property in agreement with general symmetry rules. Here, we present an extensive analysis, experimental and theoretical, of the isotropic remnant of this overtone. The isotropic spectrum turned out to be 45 times less intense than its anisotropic counterpart and to have a moment that is 350 times smaller than the moment of the anisotropic spectrum, in excellent agreement with theoretical predictions. Once the measured intensity (along with other data exclusively experimental) was fed back into the formula of the moment, a value for the $\mathrm{CO}_{2}$ mean-polarizability asymmetric stretch derivative $\partial^{2} \bar{\alpha} / \partial q_{3}^{2}$ was returned that matches the best $a b$ initio prediction to better than $4 \%$. Agreement, in order of magnitude, was found between the intensity reported herein and that reported in the sole prior study of this overtone [G. Tejeda, B. Maté, and S. Montero, J. Chem. Phys. 103, 568 (1995)]. @ 2011 American Institute of Physics. [doi:10.1063/1.3557820]
\end{abstract}

\section{INTRODUCTION}

In the jargon of spectroscopy, overtones are line spectra which, in a way analogous to sound waves, are generated at frequencies that are multiples of the fundamental frequency. In the harmonic approximation it can be shown that overtones are forbidden in both infrared and Raman spectra. However, when anharmonicity is taken into account the transitions are weakly allowed. ${ }^{1}$ Overtones have been studied with Raman spectroscopy, but little, even though spectroscopists have long been aware of their occurrence. The reason is most probably the extreme feebleness of their signals, whose intensity can be millions of times weaker than that of the corresponding fundamental transitions. To give an idea of how small the intensity of an overtone can be, as compared to the fundamental transition, consider this: in methane, the signal of the $v_{1}$ stretch vibration, recorded by our Raman experiment, was $70,000,000$ counts per second, but for the $2 v_{1}$ overtone of methane the recorded signal was only 20 counts per second. Nevertheless, this tiny signal was equally reliable and the statistical error negligibly small, while systematic errors were kept low amounting to a few percent only.

Overtones in Raman spectra have been examined only via high-resolution techniques so far. Starting in the 1950s with the pioneering works of Stoicheff, ${ }^{2-5}$ the degree of development of high-resolution experiments has witnessed rapid growth in the last fifty years. Since then, many technological innovations were accomplished that allowed scientists to extensively investigate the $\mathrm{CO}_{2}$ doublet $^{6-10} v_{1}: 2 v_{2}^{0}$ and triplet ${ }^{10}$ $2 v_{1}: 4 v_{2}^{0}: v_{1}+2 v_{2}^{0}$ Fermi resonances, and (to a much lesser extent) its $2 v_{3}$ overtone. $^{10}$

a)Electronic mail: michel.chrysos@univ-angers.fr
The central aim of high-resolution spectroscopy is the measurement of very accurate rotational constants in nonpolar molecules, mainly for the purpose of determining molecular geometries and bond lengths. High-resolution experiments allow for measurements at resolutions of $\sim 10^{-3} \mathrm{~cm}^{-1}$, so they present a clear advantage over typical incoherent Raman experiments. Although this is an unequalled level of precision and can by no means be compared to the resolutions of our experiment, which hardly attain $\simeq 0.5 \mathrm{~cm}^{-1}$, in our equipment all the effort is put into sensitivity. As has repeatedly been stressed, ${ }^{11-14}$ our experiment has been optimized especially for the detection and processing of signals as low as only few photoelectrons per week. Such a high performance mainly intends to deepen the understanding of collision-induced light scattering processes, focusing especially on relevant far wings of spectra. We recall that the latter processes arise from the loose intermolecular interactions in ensembles of molecules (gases), so they give rise to weak band spectra; this is to be contrasted with the standard Raman processes, which are processes due mainly to intramolecular interactions. ${ }^{15,16}$ It is Welsh ${ }^{17}$ who first revolutionized the area of collision-induced processes, with the observation of forbidden absorption line spectra in compressed oxygen. Since then, the area saw a tremendous boost, with an almost interminable list of contributions by distinguished scientists (see, for instance, Frommhold's or Tabisz's classic treatises, ${ }^{18-20}$ and references therein, or some selected papers in chronological order ${ }^{21-25}$ ), and more recently with experimental works by our group. ${ }^{11-14,26,27}$

In a very recent publication, ${ }^{28}$ we showed that $2 v_{3}$ is a near fully depolarized overtone, with an integrated depolarization ratio $\eta_{\text {int }}$ amounting to $6 / 7.16$, in agreement with Atkins' symmetry rule. ${ }^{29}$ This is what came out of a 
careful experimental analysis of gaseous carbon dioxide at room temperature $(T=294.5 \mathrm{~K})$, under various conditions of gas pressure. Quantitative agreement with spectral moment theory was found after incorporating electro-optical and mechanical anharmonicity effects and intermode couplings involving symmetrical and asymmetrical stretching vibrations.

In the present article, we turn our attention to the isotropic component of this band, under the same conditions of temperature and pressure. A quick look at elementary spectral theory shows that this spectrum should be extremely weak, a result attested immediately after the simple formula

$$
S^{\text {iso }}=S_{\perp}-\frac{7}{6} S_{\|}
$$

along with the definition $\eta=S_{\|} / S_{\perp}$ for the depolarization ratio. (Here, $S_{\perp}$ and $S_{\|}$refer to scattering signals, calibrated in absolute units of $\mathrm{cm}^{3}$ amagat, for a polarization of the incident beam that is perpendicular and parallel to the scattering plane, respectively.) In fact, the zeroth-order moment for the isotropic spectrum is readily expressed as a function of the corresponding anisotropic moment through the expression ${ }^{30}$

$$
M_{0}^{\text {iso }}=\frac{2}{15}\left(\eta_{\text {int }}^{-1}-\frac{7}{6}\right) M_{0}^{\text {ani }},
$$

where $\eta_{\text {int }}$ is the integrated depolarization ratio. According to our recent study, ${ }^{28} M_{0}^{\text {ani }}=3.2 \times 10^{-3}$ a.u. and $\eta_{\text {int }}=6 / 7.16$, so Eqs. (1) and (2) result in $M_{0}^{\text {iso }}=1 \times 10^{-5}$ a.u., which is a value about 300 times smaller than the value of $M_{0}^{\text {ani }}$. Although tiny, the value of $M_{0}^{\text {iso }}$ is evidence for the existence of an isotropic spectrum.

In what follows, the isotropic spectrum is extracted from accurate components for the parallel and perpendicular incident field polarization. The results are compared with theoretical predictions developed also herein, which, once again, take into account electro-optical and mechanical anharmonicity and intermode coupling between the two stretching vibrations $v_{1}$ and $v_{3}$. Comparison is made with the only existing study on this subject, published fifteen years ago by Tejeda, Maté, and Montero. ${ }^{10}$ Their value for the integrated intensity is in agreement with our value as regards the order of magnitude but differs by a factor of 3 .

\section{EXPERIMENT}

\section{A. The polarization components}

The experiment was carried out using our sensitive scattering equipment. An exhaustive description of this equipment can be found elsewhere. ${ }^{12}$ Technical information about the specific experiment on $2 v_{3}$ has been given in our preceding article ${ }^{28}$ and will be left out of the present one. The center of the $2 v_{3}$ overtone was seen at $4673 \mathrm{~cm}^{-1}$ and the band was recorded over a wide frequency interval, ranging from 4515 to $4810 \mathrm{~cm}^{-1}$.

Two independent scattering signal components, $S_{\|}$and $S_{\perp}$, were recorded as a function of Raman shift $\nu$, depending on whether the electric field of the incident beam was polarized in the direction parallel or perpendicular to the scattering plane. Then, the signals were calibrated and expressed in absolute units ( $\mathrm{cm}^{3}$ amagat). Figures 1 and 2 illustrate $S_{\|}$and $S_{\perp}$

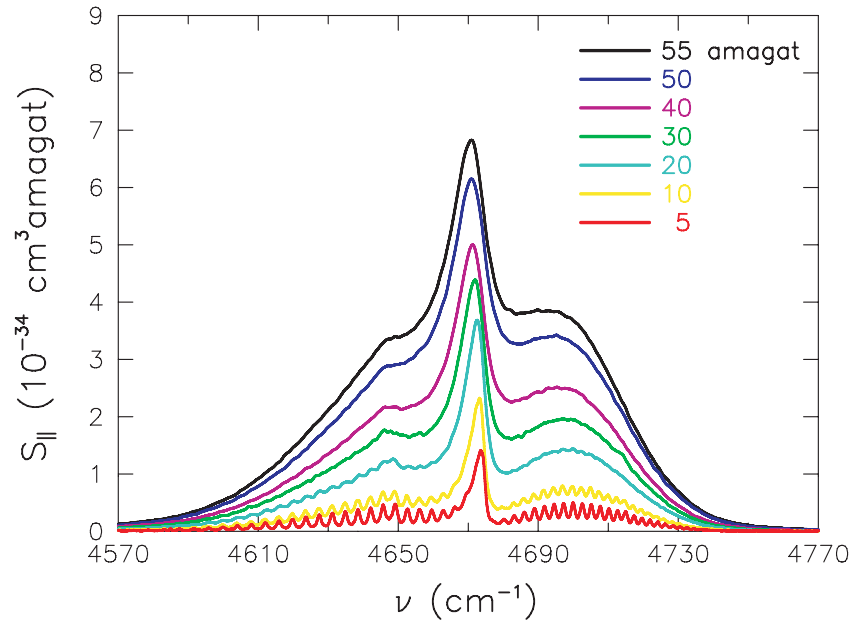

FIG. 1. Absolute-calibrated signal component $S_{\|}$(in units of $\mathrm{cm}^{3}$ amagat) as a function of frequency shift $v$ (in units of $\mathrm{cm}^{-1}$ ), for values of gas density ranging from 5 to 55 amagat (in the upward direction). The temperature is $294.5 \mathrm{~K}$.

(in units of $\mathrm{cm}^{3}$ amagat) as a function of $v$ (in units of $\mathrm{cm}^{-1}$ ), respectively, for several values of gas density ranging from 5 to 55 amagat. The small dispersion of the points is a measure of the quality of the measurements. By contrast with the rotational structure, which is visible clearly only at the lower gas densities, the pronounced structure of the three $\mathrm{O}, \mathrm{Q}$, and $\mathrm{S}$ branches is seen to persist at any density and in both spectra.

\section{B. The isotropic spectrum}

The calibrated isotropic spectrum $S^{\text {iso }}$ was deduced from the two recorded components $S_{\|}$and $S_{\perp}$, by means of the expression

$$
S^{\text {iso }}=1.017 S_{\perp}-1.184 S_{\|} .
$$

This expression accounts for the finite aperture of the scattered beam, in replacement of the ideal zero-aperture expression of Eq. (1).

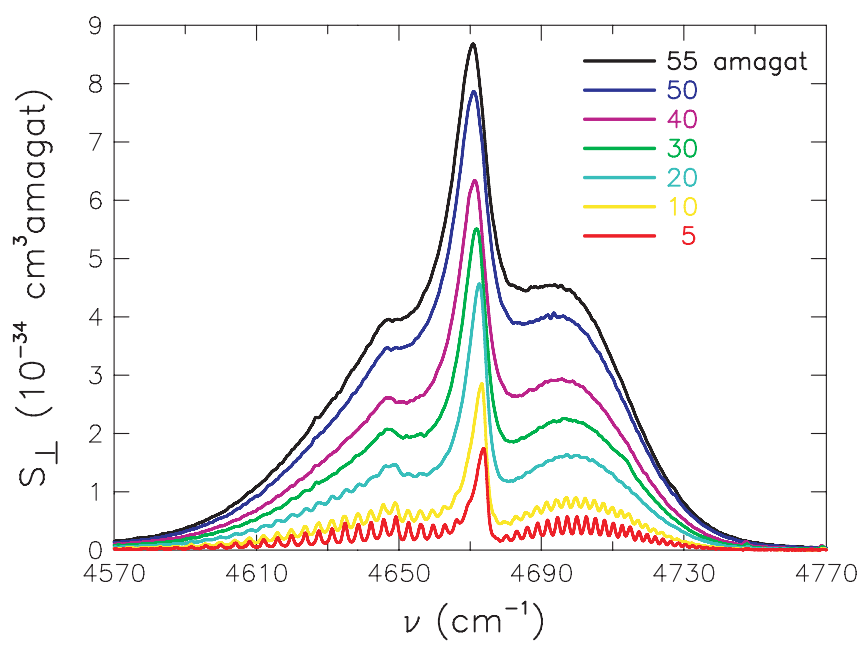

FIG. 2. Same as in FIG 1 but for $S_{\perp}$. 


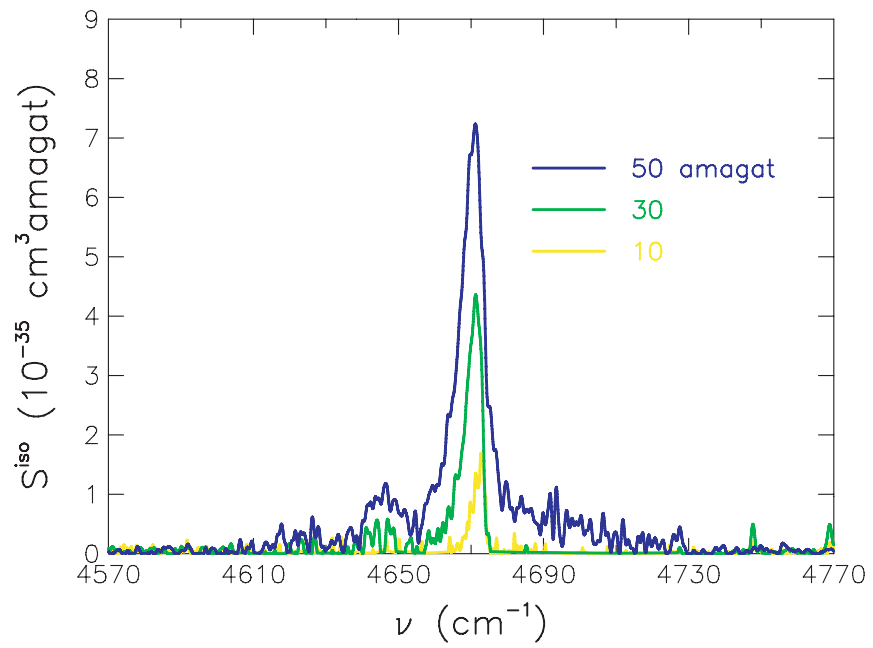

FIG. 3. Absolute-calibrated isotropic spectrum $S^{\text {iso }}$ (in units of $\mathrm{cm}^{3}$ amagat) as a function of Raman frequency shift $v$ (in units of $\mathrm{cm}^{-1}$ ), for a gas density of 10, 30, and 50 amagat (in the upward direction). The temperature is $294.5 \mathrm{~K}$.

Figure 3 shows $S^{\text {iso }}$, in units of $\mathrm{cm}^{3}$ amagat, as a function of Raman frequency shift (in units of $\mathrm{cm}^{-1}$ ) for three representative values of $\mathrm{CO}_{2}$ density, i.e., 10, 30, and 50 amagat. By contrast with the individual components $S_{\|}$and $S_{\perp}$, and with the depolarized spectrum (which is essentially the $S_{\|}$component alone), the isotropic spectrum $S^{\text {iso }}$ has neither $\mathrm{O}$ nor S branch. A well-defined Q branch is seen, whose integrated intensity turned out to scale with gas density strictly linearly. The rotational structure of the $\mathrm{Q}$ branch was not perceptible even at the lower gas densities.

To make sure that the recorded spectrum was indeed due to transitions by single molecules, a rigorous study protocol was established, enabling us to determine the exact way in which the gas density, $\rho$, affects the integrated signals $\left(S_{\|}\right)_{\text {int }}$ and $\left(S_{\perp}\right)_{\text {int }}$. According to the virial expansion, both these signals can be written as power series of $\rho$, whatever the conditions of gas pressure or temperature, so transitions that occur in single molecules generate spectra that scale linearly with $\rho$. Figure 4 illustrates $\left(S_{\|}\right)_{\text {int }}$ and $\left(S_{\perp}\right)_{\text {int }}$ as a function of $\rho$. As seen in this figure, a behavior that is strictly linear is observed over the entire range of variation in $\rho$. This is compelling evidence that the isotropic spectrum indeed comes from isolated molecules alone. The slope of the two lines defines the corresponding scattering cross sections at the zero-density limit.

\section{The spectral moment}

The zeroth-order isotropic moment was calculated by integrating $I^{\text {iso }}\left(=1.017 I_{\perp}-1.184 I_{\|}\right)$, where $I_{\|, \perp}=S_{\|, \perp} / \rho$, by means of the formula

$$
M_{0}^{\text {iso }}=\left[2 \pi\left(v_{0}-v_{s}\right)\right]^{-4} \int_{-\infty}^{+\infty} I^{\text {iso }}(v) d v .
$$

In the latter expression, $v_{0}$ denotes the laser wavenumber and $v_{s}$ the wavenumber at the center of the recorded band. The result is in $a_{0}^{6}$ units ( $a_{0}$ is the Bohr radius).

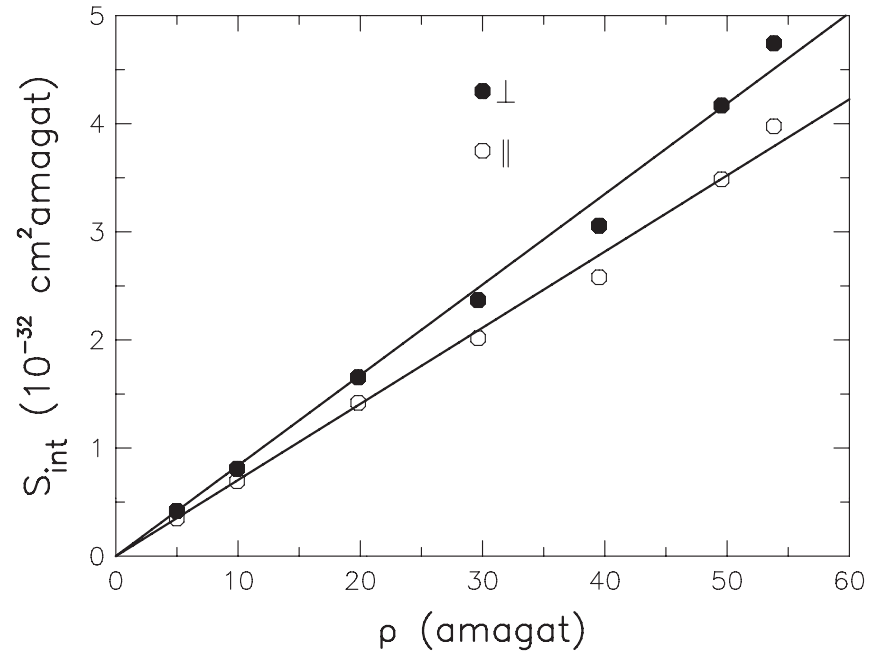

FIG. 4. Frequency-integrated spectra $\left(S_{\|, \perp}\right)_{\text {int }}$ (in units of $\mathrm{cm}^{2}$ amagat) as a function of gas density $\rho$ (in amagat units). The temperature is 294.5 K. The strictly linear behavior in both spectra shows that what we observed was indeed signals by isolated $\mathrm{CO}_{2}$ molecules.

We obtain the value $M_{0}^{\text {iso }}(\operatorname{expt})=9.1(6) \times 10^{-6} a_{0}^{6}$. This value is very close to the rough prediction of $1 \times 10^{-5} a_{0}^{6}$ that was made in the opening section of this article. In what follows, zeroth-order spectral moment theory is used to analyze the spectrum and to interpret the experiment. To this end, intermode coupling, mechanical and electrooptical anharmonicity, and high-quality $a b$ initio data for the polarizability derivatives had to be considered.

\section{THEORY}

\section{A. The transition matrix element}

The polarizability tensor, $\hat{\alpha}$, depends on nuclear coordinates and thus on normal coordinates $q_{k}, q_{l} \ldots$. Any of its components is a function of the latter coordinates, so it can be expanded into a Taylor series around the equilibrium position " $e$ ". 31 Upon using this expansion along with perturbation theory and then retaining terms up to the second order in $q_{k} q_{l} \ldots$, the transition matrix element of the polarizability's mean component, $\bar{\alpha}=\frac{1}{3} \operatorname{Tr} \hat{\alpha}$, reads ${ }^{32}$

$$
\begin{aligned}
\left\langle v_{l},\right. & \left.v_{j}, v_{i}+2|\bar{\alpha}| v_{l}, v_{j}, v_{i}\right\rangle \\
= & \frac{1}{2}\left[\left(v_{i}+1\right)\left(v_{i}+2\right)\right]^{1 / 2}\left\{\frac{1}{2} \bar{\alpha}_{i i}^{\prime \prime}+\bar{\alpha}_{i}^{\prime} \frac{k_{i i i}}{\omega_{i}}\right. \\
& \left.+\frac{1}{2} \sum_{k \neq i}\left[\bar{\alpha}_{k}^{\prime} k_{i i k}\left(\frac{1}{2 \omega_{i}-\omega_{k}}-\frac{1}{2 \omega_{i}+\omega_{k}}\right)\right]\right\} .
\end{aligned}
$$

Symbols $v_{l}, v_{j}$, and $v_{i}$ stand for vibrational quantum numbers of modes $l, j$, and $i$. Quantities $\bar{\alpha}_{i}^{\prime}$ $\left(=\partial \bar{\alpha} / \partial q_{i}\right)$ and $\bar{\alpha}_{i i}^{\prime \prime}\left(=\partial^{2} \bar{\alpha} / \partial q_{i}^{2}\right)$ denote first and second derivatives with respect to normal coordinate $q_{i}$, respectively. $\omega_{i}$ is the fundamental frequency of normal vibration $i, k_{i i i}$ denotes the cubic force constant of $i$, and $k_{i i k}$ couples the vibrations $i$ and $k$. The sum at the rhs of Eq. (5) is over all the fundamental vibrations. According to Eq. (5), the transition amplitude depends on the second derivative $\bar{\alpha}_{i i}^{\prime \prime}$ 
(electro-optical anharmonicity) and, because of anharmonic effects, on first derivatives $\bar{\alpha}_{k}^{\prime}(k \neq i)$ too.

In the specific case of the $2 v_{3}$ overtone, the only characteristics of relevance are the ones with $i=3$ and $k=1$ and the normal coordinates $q_{1}\left(\in \Sigma_{g}^{+}\right)$and $q_{3}\left(\in \Sigma_{u}^{+}\right)$. By reasons of molecular symmetry, all first derivatives of $\bar{\alpha}$, except $\bar{\alpha}_{1}^{\prime}$, are dropped in Eq. (5), so $\left\langle 00^{0} 2|\bar{\alpha}| 00^{0} 0\right\rangle$ reads

$$
\left\langle 00^{0} 2|\bar{\alpha}| 00^{0} 0\right\rangle=\frac{1}{\sqrt{2}}\left(\frac{1}{2} \bar{\alpha}_{33}^{\prime \prime}+\bar{\alpha}_{1}^{\prime} k_{331} \frac{\omega_{1}}{4 \omega_{3}^{2}-\omega_{1}^{2}}\right) .
$$

\section{B. The normal coordinates}

\section{The mode $v_{1}$}

The symmetrical vibration of $\mathrm{CO}_{2}$ can be described in a way similar to the vibration of a diatomic molecule with bond length $r=2 R$ and reduced mass $\mu=m_{\mathrm{O}} / 2$, where $m_{\mathrm{O}}$ is the atomic mass of oxygen. At the equilibrium separation, the bond length is $r=r_{e}\left(=2 R_{e}\right)$, where $R_{e}\left(=R_{\mathrm{c}-\mathrm{o}}\right.$ $\left.=2.192 a_{0}\right)^{33}$ is the length of the $\mathrm{C}-\mathrm{O}$ bond at equilibrium. With these notations, we obtain $q_{1}=2 \Delta_{1} R \sqrt{\mu \omega_{1} / \hbar}$, where $\Delta_{1} R=R-R_{e}$. Upon use of the definition of the rotational constant $B=\hbar^{2} / 2 \mu r_{e}^{2}$, we obtain

$$
\Delta_{1} R=R_{e} q_{1} \sqrt{\frac{2 B}{\hbar \omega_{1}}} .
$$

\section{The mode $v_{3}$}

For asymmetrical stretching, the normal coordinate is $\Delta_{3} R=R_{1}-R_{2},{ }^{33}$ where $R_{1}$ and $R_{2}$ denote the elongations of the two $\mathrm{C}-\mathrm{O}$ bonds.

According to Ref. 34, the quantum coordinate $q_{3}$ reads

$$
q_{3}=\frac{R_{1}-R_{2}}{2 b}=\frac{\Delta_{3} R}{2 b},
$$

where $b=0.0401 \AA=7.5778 \times 10^{-2} a_{0}$.

\section{The polarizability derivatives}

\section{The mode $v_{1}$}

In the case of the symmetrical vibration, coupled cluster calculations with single, double and partially triple excitations $[\operatorname{CCSD}(\mathrm{T})]$, done by Maroulis with a carefully designed basis set, ${ }^{35}$ result in a power expansion of the mean polarizability $\bar{\alpha}$ with the linear term equal to

$$
\bar{\alpha}(R)-\bar{\alpha}\left(R_{e}\right) \approx 13.81 \Delta_{1} R .
$$

With the help of Eq. (7), one gets

$$
\bar{\alpha}_{1}^{\prime} \approx 0.7254 \text { a.u. }
$$

We point out that this $\bar{\alpha}_{1}^{\prime}$ value, predicted $a b$ initio by Maroulis, ${ }^{35}$ is very close to the measured value $\bar{\alpha}_{1}^{\prime}$ $=12.43(7) \times 10^{-42} \mathrm{CV}^{-1} \mathrm{~m}^{2}=0.754(4)$ a.u. (Ref. 36) reported by Tejeda et al. ${ }^{10}$
TABLE I. Input data needed in Eq. (6). Polarizability derivatives are in atomic units (a.u.); vibrational frequencies and couplings are in $\mathrm{cm}^{-1}$.

\begin{tabular}{ccccc}
\hline \hline$\omega_{1}$ & $\omega_{3}$ & $\bar{\alpha}_{1}^{\prime}$ & $\bar{\alpha}_{33}^{\prime \prime}$ & $k_{331}$ \\
\hline 1353.8 & 2396.5 & $\begin{array}{l}0.754^{\mathrm{a}} \\
0.725^{\mathrm{d}}\end{array}$ & $0.0156^{\mathrm{b}}$ & $-256.3579^{\mathrm{c}}$ \\
& & & & \\
\hline
\end{tabular}

${ }^{\mathrm{a}}$ Expt., Ref. 10 .

${ }^{\mathrm{b}} \operatorname{CCSD}(\mathrm{T})$, Ref. 33.

${ }^{\mathrm{c}}$ Reference 37.

${ }^{\mathrm{d}} \operatorname{CCSD}(\mathrm{T})$, Ref. 35 .

\section{The mode $v_{3}$}

In the case of the asymmetrical vibration, $\operatorname{CCSD}(\mathrm{T})$ calculations ${ }^{33}$ provide

$$
\bar{\alpha}\left(\Delta_{3} R\right)-\bar{\alpha}(0) \approx 0.34\left(\Delta_{3} R\right)^{2},
$$

so the second derivative of the mean polarizability is

$$
\bar{\alpha}_{33}^{\prime \prime} \approx 0.0156 \text { a.u. }
$$

Table I gathers all the input data that were needed to calculate the transition matrix element of Eq. (6). Polarizability derivatives are given in atomic units (a.u.); vibrational frequencies for the two modes and their coupling are in $\mathrm{cm}^{-1}$.

\section{The spectral moment}

Upon use of Eq. (6) and of the ab initio parameter values of Table I, $\left\langle 00^{0} 2|\bar{\alpha}| 00^{0} 0\right\rangle$ reads

$$
\begin{aligned}
\left\langle 00^{0} 2|\bar{\alpha}| 00^{0} 0\right\rangle & =\frac{1}{\sqrt{2}}\left(7.8095 \times 10^{-3}-11.909 \times 10^{-3}\right) \\
& =-2.899 \times 10^{-3} \text { a.u. }
\end{aligned}
$$

Obviously, the sign of the intermode coupling is opposite to that of the $\frac{1}{2} \bar{\alpha}_{33}^{\prime \prime}$ term and its magnitude exceeds by $50 \%$ the magnitude of $\frac{1}{2} \bar{\alpha}_{33}^{\prime \prime}$. It is precisely this subtle interplay between the bare $\frac{1}{2} \bar{\alpha}_{33}^{\prime \prime}$ term and the "coupling correction" that governs the isotropic scattering process and determines $S^{\text {iso }}$. Any absence of consideration of this coupling would draw meaningless conclusions. obtain

Using the expression ${ }^{38} M_{0}^{\text {iso }}=\left|\left\langle 00^{0} 2|\bar{\alpha}| 00^{0} 0\right\rangle\right|^{2}$, we

$$
M_{0}^{\text {iso }}(\text { th })=8.4 \times 10^{-6} a_{0}^{6} .
$$

This theoretical value (th) matches our measured one $\left[M_{0}^{\text {iso }}(\right.$ expt $\left.)=9.1(6) \times 10^{-6} a_{0}^{6}\right]$ to better than $8 \%$.

Table II gathers our moment values, along with the value measured and reported in the sole prior work of this overtone. For comparison, moment values for the anisotropic spectrum are also shown.

\section{E. The second polarizability derivative}

In this paragraph, we intend to deduce a value for the property $\partial^{2} \bar{\alpha} / \partial q_{3}^{2}$ from data that are exclusively experimental. To do this, we will now proceed in the reverse order: Upon 
TABLE II. Moment values obtained experimentally and theoretically for the isotropic spectrum. The values for the anisotropic spectrum are shown for comparison.

\begin{tabular}{lcc}
\hline \hline & Theory & expt \\
\hline$M_{0}^{\text {iso }}\left(\times 10^{-6}\right.$ a.u. $)$ & $8.4^{\mathrm{a}}$ & $9.1(6)^{\mathrm{a}}$ \\
& & $30(10)^{\mathrm{b}}$ \\
$M_{0}^{\text {ani }}\left(\times 10^{-3}\right.$ a.u. $)$ & $3.53^{\mathrm{c}}$ & $3.20(15)^{\mathrm{c}}$ \\
\hline \hline
\end{tabular}

a This work.

${ }^{\mathrm{b}}$ Reference 10. For unit conversion, see Ref. 39.

${ }^{c}$ Reference 28.

assuming $\left\langle 00^{0} 2|\bar{\alpha}| 00^{0} 0\right\rangle= \pm \sqrt{M_{0}^{\text {iso }}(\operatorname{expt})}$ and feeding the resulting values back into Eq. (6), we obtain

$$
\bar{\alpha}_{33}^{\prime \prime}=2\left( \pm \sqrt{2 M_{0}^{\text {iso }}(\operatorname{expt})}-\bar{\alpha}_{1}^{\prime} k_{331} \frac{\omega_{1}}{4 \omega_{3}^{2}-\omega_{1}^{2}}\right) .
$$

Then, by employing the experimental value ${ }^{10}$ for the parameter $\bar{\alpha}_{1}^{\prime}$ (see Table I), we get

$$
\bar{\alpha}_{33}^{\prime \prime}=\left\{\begin{array}{l}
0.0333 \text { a.u. } \\
0.0162 \text { a.u., }
\end{array}\right.
$$

with the upper (lower) value corresponding to the $+(-)$ sign of $\pm \sqrt{M_{0}^{\text {iso }}(\operatorname{expt})}$.

Interestingly, the lower value shown above matches the $a b$ initio value $e^{33}$ of Table I to better than $4 \%$. This accuracy exceeds typical accuracies for second polarizability derivatives, which are often inaccurate by a factor of $2 .{ }^{40}$

Table III gathers values for $\bar{\alpha}_{33}^{\prime \prime}$ that we obtained with use of data exclusively experimental, by means of Eq. (13) and of the assumption $\left\langle 00^{0} 2|\bar{\alpha}| 00^{0} 0\right\rangle= \pm \sqrt{M_{0}^{\text {iso }}(\text { expt })}$. As input values for $M_{0}^{\text {iso }}($ expt), the value measured by Tejeda et al. ${ }^{10}$ was used as well as our own measurement. For the sake of comparison, the ab initio value is also shown. ${ }^{33} \mathrm{Up}-$ per entries correspond to the spurious solution of the equation $x^{2}-M_{0}^{\text {iso }}(\operatorname{expt})=0$.

Whereas the value 0.0162(3) agrees to better than $4 \%$ with the $a b$ initio value of Maroulis, disagreement by more than $70 \%$ is found between the value 0.0091(2) and the $a b$ initio prediction.

A practical means of quantifying the relative importance of the intermode coupling in $2 \nu_{3}$ is to measure the degree of deviation between the two ratios $\chi^{\text {no }}=\left|\beta_{33}^{\prime \prime} / \bar{\alpha}_{33}^{\prime \prime}\right|$ and $\chi^{\text {on }}$

TABLE III. Values for $\bar{\alpha}_{33}^{\prime \prime}$ obtained upon use of Eq. (13) and of data that are exclusively experimental. For $M_{0}^{\text {iso }}$ (expt), use of our measurement and of the measurement reported by Tejeda $e t$ al. was made. The ab initio value is shown for comparison ${ }^{33}$. The entries in the upper row are spurious solutions.

\begin{tabular}{lccc}
\hline \hline (a.u.) & $\operatorname{expt}^{\mathrm{a}}$ & $\operatorname{expt}^{\mathrm{b}}$ & $\operatorname{CCSD}(\mathrm{T})^{\mathrm{c}}$ \\
\hline & $0.0404(2)$ & $0.0333(3)$ & \\
$\bar{\alpha}_{33}^{\prime \prime}$ & $0.0091(2)$ & $0.0162(3)$ & 0.0156 \\
\hline \hline
\end{tabular}

${ }^{\mathrm{a}}$ Reference 10 .

${ }^{\mathrm{b}}$ This work.

${ }^{\mathrm{c}}$ Reference 33
$=\sqrt{M_{0}^{\text {ani }}(\operatorname{expt}) / M_{0}^{\text {iso }}(\operatorname{expt})}$. (The superfix "on" denotes that the intermode coupling was switched on in the calculation; "no" denotes that no intermode coupling was considered.) Whereas in the absence of coupling between the modes $\nu_{1}$ and $\nu_{3}$ both those quotients should equal $\left|\left\langle 00^{0} 2|\beta| 00^{0} 0\right\rangle /\left\langle 00^{0} 2|\bar{\alpha}| 00^{0} 0\right\rangle\right|$, here, two strikingly different values were obtained: $\chi^{\text {no }}=8.6, \chi^{\text {on }}=19$. The last value is particularly large, a result that evidences how strong intermode effects are for this band.

The sole fact that $\chi \gg 1$ is evidence enough that the band is strongly depolarized. Yet, the value of $\eta_{\text {int }}\left[=6 \chi^{2}\left(7 \chi^{2}\right.\right.$ $+45)^{-1}$ ] was greatly enhanced upon inclusion of the intermode coupling, changing from $\eta_{\text {int }}^{\text {no }}=0.79$ to $\eta_{\text {int }}^{\text {on }}=0.84$. Undoubtedly, accounting for this coupling is getting a notch closer to the upper bound for the depolarization ratio $\left(\eta_{\max }\right.$ $=6 / 7$ ), in striking agreement with our experiment (see also Fig. 3 in Ref. 28).

\section{SYNOPSIS}

This article reports and analyzes the isotropic Raman spectrum of the $2 v_{3}$ overtone band of $\mathrm{CO}_{2}$. The measurements were taken with a high-sensitivity incoherent Raman scattering equipment, in the frequency region $4500-4800 \mathrm{~cm}^{-1}$, for two orthogonal field-polarization arrangements of the incident beam. High purity gas samples were used, at $T=294.5$ $\mathrm{K}$, and at densities ranging from 5 to 55 amagat. As was borne out from our measurements, the moment of the isotropic spectrum is 350 times smaller than that of its anisotropic counterpart $^{28}$ and its integrated intensity 45 times smaller than the integrated intensity of that spectrum. This finding is in excellent agreement with theoretical predictions, provided that mechanical or electro-optical anharmonicity and couplings were incorporated via measurements ${ }^{10}$ or ab initio data, ${ }^{33,35}$ and that the heavy mixing between $v_{1}$ and $v_{3}$ modes was considered properly to account for the subtle interplay between competing contributions. Once fed back into the formulas, the measured intensity turned out to generate a value for the mean-polarizability second stretch derivative, $\partial^{2} \bar{\alpha} / \partial q_{3}^{2}$, that matches the best ab initio prediction ${ }^{33}$ to better than $4 \%$.

${ }^{1}$ S. Califano, Vibrational states (Wiley, New York, 1976).

${ }^{2}$ B. P. Stoicheff, Can. J. Phys. 35, 730 (1957).

${ }^{3}$ B. P. Stoicheff, Can. J. Phys. 36, 218 (1958).

${ }^{4}$ B. P. Stoicheff, in Advances in Spectroscopy, edited by H. W. Thompson, (Wiley-Interscience, New York, 1959), Vol. 1, pp. 91-174.

${ }^{5}$ A. Weber, in The Raman Effect, edited by A. Anderson, (Marcel Dekker, New York, 1973), Vol. 2, pp. 543-757.

${ }^{6}$ H. Finsterhölzl, H. W. Klöckner, K. Srinivasan, H. W. Schrötter, and J. Brandmüller, Indian J. Pure Appl. Phys. 16, 370 (1978).

${ }^{7}$ H. W. Klöckner, H. Finsterhölzl, K. Srinivasan, and H. W. Schrötter, Appl. Spectrosc. 32, 401 (1978).

${ }^{8}$ H. Finsterhölzl, Ber. Bunsenges. Phys. Chem. 86, 797 (1982).

${ }^{9}$ P. Wienecke, H. Finsterhölzl, H. W. Schrötter, and J. Brandmüller, Appl. Spectrosc. 40, 70 (1986).

${ }^{10}$ G. Tejeda, B. Maté, and S. Montero, J. Chem. Phys. 103, 568 (1995).

${ }^{11}$ F. Rachet, M. Chrysos, C. Guillot-Noël, and Y. Le Duff, Phys. Rev. Lett. 84, 2120 (2000).

${ }^{12}$ F. Rachet, Y. Le Duff, C. Guillot-Noël, and M. Chrysos, Phys. Rev. A 61, 062501 (2000).

${ }^{13}$ M. Chrysos, S. Dixneuf, and F. Rachet, Phys. Rev. A 80, 054701 (2009). 
${ }^{14}$ I. A. Verzhbitskiy, M. Chrysos, F. Rachet, and A. P. Kouzov, Phys. Rev. A 81, 012702 (2010); M. Chrysos and I. A. Verzhbitskiy, Phys. Rev. A 81, 042705 (2010); I. A. Verzhbitskiy, M. Chrysos, and A. P. Kouzov, Phys. Rev. A 82, 052701 (2010).

${ }^{15}$ T. Bancewicz and S. Kielich, J. Chem. Phys. 75, 107 (1981).

${ }^{16}$ Useful in the understanding of the symmetry concepts treated in the present or in the preceding paper (Ref. 28) was the analysis reported by T. Bancewicz and S. Kielich (Ref. 15), about the collision-induced mechanisms in Raman scattering for nontotally symmetric vibrations.

${ }^{17}$ M. F. Crawford, H. L. Welsh, and J. L. Locke, Phys. Rev. 75, 1607 (1949).

${ }^{18}$ L. Frommhold, Adv. Chem. Phys. 46, 1 (1981).

${ }^{19}$ L. Frommhold, Collision-induced Absorption in Gases (Cambridge University Press, Cambridge, England, 1993).

${ }^{20}$ Collision- and Interaction-Induced Spectroscopy, edited by G. C. Tabisz and M. N. Neuman (Kluwer, Dordrecht, 1995).

${ }^{21}$ J. P. McTague and G. Birnbaum, Phys. Rev. Lett. 21, 661 (1968).

${ }^{22}$ J. C. Lewis and J. Van Kranendonk, Phys. Rev. Lett. 24, 802 (1970).

${ }^{23}$ A. Borysow and L. Frommhold, Adv. Chem. Phys. 75, 439 (1989).

${ }^{24}$ L. Ulivi, N. Meinander, and F. Barocchi, Phys. Rev. Lett. 75, 3094 (1995).

${ }^{25}$ M. Zoppi, L. Ulivi, M. Santoro, M. Moraldi, and F. Barrochi, Phys. Rev. A 53, R1935 (1996).

${ }^{26}$ Y. Le Duff, Phys. Rev. Lett. 90, 193001 (2003).

${ }^{27}$ Y. Le Duff, J. Chem. Phys. 119, 1893 (2003).

${ }^{28}$ M. Chrysos, I. A. Verzhbitskiy, F. Rachet, and A. P. Kouzov, J. Chem. Phys. 134, 044318 (2011).

${ }^{29}$ P. Atkins and J. de Paula, Atkins' Physical Chemistry, 8th ed. (Oxford University Press, UK, 2006). According to a general symmetry rule cited therein, p. 464, "only totally symmetrical vibrations give rise to polarized lines in which the incident polarization is largely preserved" and "vibrations that are not totally symmetrical give rise to depolarized lines."

${ }^{30}$ Proof: $M_{0}^{\text {iso }} /\left(\frac{2}{15} M_{0}^{\text {ani }}\right)=\int_{-\infty}^{\infty} S^{\text {iso }} d v / \int_{-\infty}^{\infty} S^{\text {ani }} d v=\int_{-\infty}^{\infty}\left(S_{\perp}-\frac{7}{6} S_{\|}\right) d v$ $/ \int_{-\infty}^{\infty} S_{\|} d v=\int_{-\infty}^{\infty} S_{\perp} d v / \int_{-\infty}^{\infty} S_{\|} d v-\frac{7}{6}=\eta_{\text {int }}^{-1}-\frac{7}{6}$.

${ }^{31}$ D. A. Long, The Raman Effect. A Unified Treatment of the Theory of Raman Scattering by Molecules (Wiley, Chichester, UK, 2002).

${ }^{32}$ T. D. Kolomiitsova and D. N. Shchepkin in Advances in Spectroscopy, edited by R. J. H. Clark and R. E. Hester (Wiley, Chichester, 1995), Vol. 23.

${ }^{33}$ A. Haskopoulos and G. Maroulis, Chem. Phys. Lett. 417, 235 (2006).

${ }^{34}$ T. D. Kolomiitsova, A. V. Lyaptsev, and D. N. Shchepkin, Opt. and Spectrosc. 88, 648 (2000).

${ }^{35}$ G. Maroulis, Chem. Phys. 291, 81 (2003).

${ }^{36}$ Given the conversion factor (Ref. 35) $1 a_{0}^{3}=1 e^{2} a_{0}^{2} E_{h}^{-1}=1.648778$ $\times 10^{-41} \mathrm{CV}^{-1} \mathrm{~m}^{2}$, we obtain $12.43(7) \times 10^{-42} \mathrm{CV}^{-1} \mathrm{~m}^{2}=0.754(4)$ a.u.

${ }^{37}$ A. Chedin, J. Mol. Spectrosc. 76, 430 (1979).

${ }^{38}$ R. G. Gordon, J. Chem. Phys. 39, 2788 (1963).

${ }^{39}$ The value reported by Tejeda et al. (Ref. 10) is $\left|\left\langle 00^{0} 2|\bar{\alpha}| 00^{0} 0\right\rangle\right|$ $=0.091(15) \times 10^{-42} \mathrm{CV}^{-1} \mathrm{~m}^{2}$. Given the conversion factor of (Ref. 36), we obtain $\left|\left\langle 00^{0} 2|\bar{\alpha}| 00^{0} 0\right\rangle\right|=5.52(91) \times 10^{-3} a_{0}^{3}$ and thus $\left\langle 00^{0} 2|\bar{\alpha}| 00^{0} 0\right\rangle^{2}=30(10) \times 10^{-6} a_{0}^{6}$.

${ }^{40}$ It has also been pointed out by Tejeda et al. that the accuracy of second derivatives very much depends on each particular case, ranging from $10 \%$ to $100 \%$ (their words) (Ref. 10, Introduction section). 